\title{
Paraphenylenediamine (PPD) poisoning - a case report do we really know kala pathar?
}

\begin{abstract}
Paraphenylenediamine is active ingredient of most of hair dyes used by the common public because of easy availability and cost effectiveness. PPD is a derivative of p-nitroaniline which is an aromatic amine and a main constituent of many industrial chemicals. Paraphenylenediamine's toxicity is subjected to its topical application and oral ingestion. When it is used in any of above mentioned way, it produces many local and systemic side effects. Extent of toxic side effects varies with dose ingested and duration since it was taken. Local effects are swelling of airway, face, eyes and tongue, while systemic effects include dysphagia, respiratory distress, rhabdomyolysis, intravascular hemolysis, acute renal failure and cardiotoxicity. There is no particular antidote for this poisoning. Main stay of management is supportive care only. Although PPD toxicity is a life threatening emergency, however early diagnosis and prompt management of the complications can lead to better prognosis. We are reporting here a case of Hair dye poisoning of a young female who presented with features of angioedema, stridor, severe anaphylactic reaction and acute kidney injury. She was successfully managed with emergency tracheostomy and on lines of anaphylactic reaction.
\end{abstract}

Keywords: paraphenylenediamine, angioedema, anaphylactic reaction
Volume I0 Issue I - 2018

\author{
Ehsan Ahmad \\ Resident, Department of Anesthesia and Pain management, \\ Shaukat Khanum Memorial Cancer Hospital and Research \\ Centre, Pakistan
}

Correspondence: Ehsan Ahmad, Resident, Department of Anaesthesia and pain management, Shaukat Khanum Memorial Cancer Hospital and Research Centre, Lahore, Pakistan, Tel +92 345 4942626, Email ehsanahmad8 I4@gmail.com

Received: January 20, 2018 | Published: February 14, 2018

\section{Introduction}

Paraphenylenediamine is commonly used in hair dyes .It is a derivative of P-Nitroanaline and is mixed with Henna in the various regions of the world. These dyes are used to color the palms of hands, hair, soles of feet etc. Use of theses dyes leads to exposure to PPD and causes various local and systemic complications. Severity of these complications depends upon the extent of exposure, mode of exposure whether oral, inhalational or mucosal and also the content of active ingredient i.e. PPD in the dye. Content of PPD in variable in different types of dyes available in the market. Branded dyes have comparatively less content of PPD than stone hair dyes. So chances of complications are less with exposure to branded dyes.

PPD poisoning is a common but preventable occupational hazard in our part of the world. Early recognition and prompt management of manifestations is vital. Nonexistence of antidote and Life threatening complications of PPD demand from practicing physician to have high suspicion of this toxicity. Once identified, team management and targeted approach is the key for improvement of patient's survival.

\section{Case report}

A 21years old female presented in emergency room (ER) with strider and difficulty in breathing for last few hours. She was rushed to operating room (OR) for emergency tracheostomy for impeding airway obstruction due to severe angioedema of larynx. Tracheostomy was done under local anesthesia without any complications. Patient was transferred to post anesthesia care unit (PACU) after the procedure. Detailed history revealed that she was working in henna factory where they use "Kala Pathar" (in URDU language) as one of the component for henna. We didn't find any other co morbidities. After two hours in PACU, post tracheostomy patient started having tachycardia with heart rate of around 120 beats per minute, respiratory rate of 25-30 per minute, perfuse sweating and hypotension with blood pressure of $85 / 35 \mathrm{mmHg}$. Her conscious level remained appropriate. There was not any history of drug abuse.
She was fluid resuscitated with crystalloids and colloids, was given supplemental oxygen, injection dexamethasone 8mg, chlorpheniramine $50 \mathrm{mg}$, hydrocortisone $250 \mathrm{mg}$. Urinary catheter was passed and she was passing cola colored dark urine which raised suspicion of rhabdomyolosis.

Meanwhile we probed into her occupational history more and we came to know that same episode happened to her sister working in same factory resulting in her demise. A bit more into this "KALA PATHAR" highlighted PARAPHENYLENEDIAMINE (PPD), as culprit used in henna. She dropped her blood pressure to $80 / 35 \mathrm{mmHg}$ and we had to start her on nor-epinephrine infusion. Later we shifted her to intensive care unit. Her initial blood work up showed hemoglobin $12.5 \mathrm{mg} / \mathrm{dl}$, white cell count of 9.2, platelet count of 225 , urea 45 , Creatinine $2.8 \mathrm{mg} / \mathrm{dl}$, sodium 145 , potassium 5.9 , and urine examination was positive for rhabdomyolosis. Forced dieresis with diuretics and manitol was done to preserve renal function. After 4 hours with no urine output she was put on dialysis to treat her raising Creatinine and acidosis. Dialysis was done for 24 hours but her acidosis was resistant to treatment. Patient's Creatinine was creeping up to $7.8 \mathrm{mg} / \mathrm{dl}$ with $\mathrm{pH}$ of 6.8 . After around 36hours patient died of severe metabolic acidosis and multiorgan failure.

\section{Discussion}

Centre for Disease Control and prevention (CDC) lists paraphenylinediamaine as a contact allergen 1 . It is oxidized to various intermediates out of which major product formed is Bondrowski's base which is allergenic, mutagenic and highly toxic. ${ }^{1}$ In 1924 first case of PPD toxicity was reported in a hair dresser followed by hair dye handling. ${ }^{2}$ PPD exposure leads to several local and systemic manifestations making it a potential health hazard. ${ }^{3}$ Direct contact can severely irritate the skin and eyes with possible damage to vision as well. Inhalational injury can cause nose, throat, and lung irritation leading to cough, wheeze and shortness of breath. In one prospective hospital-based cohort study conducted by Mazin et al. showed that exposure to PPD was via oral route in $100 \%$ of patiets. ${ }^{4}$ There are also 
many systemic manifestations including abdominal pain, nausea, high blood pressure, dizziness, seizures and even coma. It can damage liver and kidneys too. Systemic toxicity may occur in two phases. In phase I, there is edema of neck, face and airway causing airway obstruction, vomiting and gastritis. In phase II, there is sub-acute presentation with hemolysis, rhabdomyolysis and acute renal failure. ${ }^{4}$ Occupational safety and health administration (OSHA) gives the legal airborne permissible exposure limit (PEL) as $0.1 \mathrm{mg} / \mathrm{m} 3$ averaged over 8 hours work shift. ${ }^{1}$ Exposure of employees to hazardous substances should be routinely evaluated. This may include collecting personal and area air sample for PPD levels.

Our patient developed skin rash, angioedema and dark colored urine. She had inspiratory stridor Tachypnea and dyspnea leading to respiratory insufficiency. So emergency tracheostomy was done to secure the airway.

PPD toxicity has high morbidity and mortality but early identification of the symptoms and prompt management can be lifesaving in these patients. Supportive care including airway breathing and circulation along with gastric lavage, good hydration, steroids, antihistamines and vasopressors should be considered as per requirement. ${ }^{4}$ Multimodal team approach in the management is the key. Involvement of physician, intensivist and ENT surgeon can lead to better management.

\section{Acknowledgments}

None.

\section{Conflicts of interest}

None.

\section{References}

1. Chaudhary SC, Salwani KK, Singh K. Paraphenylenediamine poisoning. Niger J Clin Pract. 2013;16(2):258-259.

2. Ashar A. Acute angioedema in paraphenylenediamine poisoning. $J$ Pak Med Assoc. 2003;53(3):120-122.

3. Ahlawat G, Garg, Kirti, et al. Methylprednisolone in hair dye poisoning. Anaesth Pain \& Intensive Care. 2014;18(1):49-51.

4. Shigidi M, Muhammed O, Ibrahim M, et al. Clinical presentation, treatment and outcome of paraphenylene-diamine induced acute kidney injury following hair dye poisoning: a cohort study. Pan Afr Med J. 2014;19:163. 